\title{
La metoclopramida no se asocia a resultados obstétricos adversos
}

Metoclopramide is not associated with adverse obstetric outcomes

Pasternak B y col, JAMA, 2013;310(15):1601-1611

\section{Objetivo}

Establecer la relación entre el uso de metoclopramida durante el embarazo y el desarrollo de anomalías congénitas, aborto espontáneo y muerte fetal intrauterina.

\section{Diseño}

Estudio de cohorte realizado a partir del Registro de Nacimientos y del Registro Nacional de Pacientes de Dinamarca, abarcando el período 1997 a 2011. Comparó mujeres embarazadas expuestas a metoclopramida con controles no expuestas (relación 1:4). Se incluyeron embarazos únicos con recién nacido vivo, muerte fetal intrauterina o aborto espontáneo mayor a seis semanas de amenorrea. Se estimó la exposición cotejando registros de prescripción médica.

\section{Medición de resultados principales}

Los casos de anomalías congénitas mayores diagnosticados dentro del primer año de vida fueron obtenidos del Registro Nacional de Pacientes y se clasificaron de acuerdo al EUROCAT (European Surveillance of Congenital Anomalies) excluyendo anomalías explicadas por otra causa (ejemplo: anomalías cromosómicas, síndromes genéticos). Se realizó control por confundidores a través del apareamiento de variables (entre expuestas y no expuestas) como la edad, el año calendario y múltiples variables.

\section{Resultados}

Se resumen en la Tabla 1.

Tabla 1. Asociación entre uso de metoclopramida durante el embarazo y resultados obstétricos

\begin{tabular}{l|c|c|c}
\multirow{2}{*}{ Resultado obstétrico } & Expuestas (28.486) & No expuestas (113.698) & \multirow{2}{*}{ OR ajustado / HR (IC 95\%) } \\
\cline { 2 - 3 } & $\begin{array}{c}\text { Casos / 1.000 nacidos vivos } \\
\text { (IC 95\%) }\end{array}$ & $\begin{array}{c}\text { Casos / 1.000 nacidos vivos } \\
\text { (IC 95\%) }\end{array}$ & OR 0,93 (0,86 a 1,02) \\
\hline Malformaciones congénitas mayores & $25,3(23,5$ a 27,1) & $26,6(25,7$ a 27,5) & HR $0,35(0,33$ a 0,38$)$ \\
\hline Aborto espontáneo & $20,0(18,5$ a 21,4) & $62,1(60,9$ a 63,3$)$ & HR $0,90(0,74$ a 1,08$)$ \\
\hline Muerte fetal & $3,5(2,9$ a 4,1) & $3,9(3,6$ a 4,2) & \\
\hline
\end{tabular}

OR: Odds ratio. HR: hazarol ratio.

\section{Conclusiones}

El uso de metoclopramida en el embarazo no se asoció con un riesgo aumentado de anomalías congénitas mayores, aborto espontáneo ni muerte fetal.
Conflicto de Interés: Los autores no reportan. Fuente de financiamiento: El estudio fue financiado por un subsidio del Danish Medical Research Council: grant 11-115854.

\section{Comentario}

En forma casi cotidiana los médicos deben enfrentarse a una variedad de situaciones en la cual deben prescribir medicamentos a una mujer, embarazada o en edad fértil, y es donde surge la duda sobre la potencial teratogenicidad. Entre las condiciones más frecuentes se encuentran las náuseas y los vómitos durante el embarazo, que afectan a un porcentaje importante de mujeres, fundamentalmente en el primer trimestre ${ }^{1,2}$. El tratamiento aprobado para este trastorno y que demostró ser efectivo en revisiones sistemáticas ${ }^{3,4,5}$ es la aplicación de vitamina B6 y el antihistamínico doxilamina, sin embargo en nuestro medio la droga más prescripta para esta indicación es la metocloprramida. La evaluación del cociente riesgo de daño: posible beneficio es un verdadero desafío. Los potenciales efectos deletéreos de una droga sobre el feto dependerán: de la naturaleza química y física, del grado de exposición fetal y de la etapa de desarrollo al momento de la exposición. La enorme dificultad con la que nos solemos encontrar es la falta de datos confiables sobre la seguridad de la droga en seres humanos (particularmente en la embarazada), ya que resulta una tarea bastante compleja traducir a la clínica los datos obtenidos en experiencias con animales. La otra fuente de información son los estudios epidemiológicos que suelen tener entre otros problemas la identificación difusa de la ventana de exposición, la caracterización inadecuada de la misma exposición, el sesgo de reporte, sesgo de evaluación de resultados, y el impacto de las intervenciones para mejorar los resultados. Este estudio logra saltear muchas de estas desventajas por utilizar registros de muy buena calidad, tener un elevado número de pacientes y realizar ajustes por confundidores comunes. Sin embargo tiene como toda investigación teratológica la debilidad de la caracterización de la exposición $n^{6}$. Por otra parte ningún teratógeno aumenta el riesgo global de malformaciones, si no que presenta un patrón definido de anomalías y al examinar las categorías individuales el número de expuestos por categoría es pequeño para encontrar diferencias significativas ${ }^{7}$.

\section{Conclusiones del comentador}

A pesar de todas las dificultades metodológicas, los estudios epidemiológicos son la mejor evidencia disponible; y dado que es imposible establecer la seguridad completa de una droga, el estudio que comentamos es una excelente pieza de información acerca de la ausencia de daño. Esto es importante ya que en nuestro medio la metoclopramida es de uso extendido entre las embarazadas, más aún durante el primer trimestre, período de organogénesis.

Gustavo Hernán Izbizky y María Eugenia Carducci [ Servicio de Obstetricia del Hospital Italiano de Buenos Aires. gustavo.izbizky@ hospitalitaliano.org.ar, maria.carducci@ hospitalitaliano.org.ar ]

Izbizky GH, Carducci ME. La metoclopramida no se asocia a resultados obstétricos adversos. Evid Act Pract Ambul. Ene-Mar 2014;17(1):24. Comentado de: Pasternak B, Svanström H, Mølgaard-Nielsen D, et al. Metoclopramide in pregnancy and risk of major congenital malformations and fetal death. JAMA. 2013;320(15):1601-1611. PMID: 24129464.

\section{Referencias}

1. Lacroix R y col. Nausea and vomiting during pregnancy: a prospective study of its frequency, intensity, and patterns of change. Am J Obstet Gynecol. 2000;182(4):931-937. 2- Niebyl JR. Clinical Practice: nausea and vomiting in pregnancy. N Engl J Med. 2010;363(16):1544-1550.

3- Matthews A, Dowswell T, Haas D, Doyle M, O'Mathúna D. Interventions for nausea and vomiting in early pregnancy. Cochrane Database of Systematic Reviews. Issue 11, 2013.

4- Festin M. Nausea and vomiting in early pregnancy. Clinical Evidence. 2009;June 3:1405.

5-Evidence-based view of safety and effectiveness of pharmacologic therapy for nausea and vomiting of pregnancy (NVP). Am J Obstet Gynecol. 2002;186(5):S256-61.

6- Koren G. Ethical framework for observational studies of medicinal drug exposure in pregnancy. Teratology. 2002;65:191-195

7- Webster W, Freeman J. Is this drug safe in pregnancy? Reprod Toxicol. 2001;15:619-629. 